\title{
Efficient Utilization of Auxiliary Information on Estimation of Population Mean Using Exponential Type Estimators in Successive Sampling
}

\author{
Surya K. Pal * \\ School of Studies in Statistics, \\ Vikram University, Ujjain, M.P., India-456010 \\ suryakantpal6676@gmail.com \\ Housila P. Singh \\ School of Studies in Statistics, \\ Vikram University, Ujjain, M.P., India-456010 \\ hpsujn@gmail.com
}

Received 12 February 2016

Accepted 25 December 2016

\begin{abstract}
This paper advocates the problem of estimating the population mean on the current occasion in two occasion successive (rotation) sampling under the transformed auxiliary variable using exponential method of estimation. Four different type estimators are suggested for estimating the current population mean in two occasion successive (rotation) sampling. Optimum replacement policies and performance of suggested estimators have been discussed. Outcomes are interpreted through empirical study.
\end{abstract}

Keywords: Auxiliary variable; Study variable; Successive sampling; Optimum replacement policy; Efficiency. 2000 Mathematics Subject Classification: 62D05.

\section{Introduction}

The information on an auxiliary variable is usually available for all the units of a finite population in sample surveys. In many sample survey studies, when the character under study of finite population changes over time, one time survey carried out on a single occasion provides information about the characteristic of the surveyed population for the given occasion and unable to give information regarding the nature of change over different occasions and the estimates of the population parameters over all occasions or on the most recent occasion. There are many problems of practical interest in social sciences in which various characters opt to change over time with respect to different parameters. Hence, one is often concerned with measuring the characteristics of a population on several occasions to estimate the trend in time of population means as a time series or current value of population mean over several points of time. For example, an investigator, or owner of the industry of cold drinks may be paying attention in the following type of problems:

(i) The average or total sale of cold drink for current season;

(ii) The change in average sale of cold drink for two different seasons;

(iii) Simultaneously to know the both (i) and (ii).

${ }^{*}$ Corresponding author. 
Or in many countries, monthly labor-force surveys are conducted to estimate the number of employed individuals and the rate of unemployment. Other examples are weekly or monthly surveys on prices of goods are conducted to determine the customer price index, and political opinion surveys are conducted at regular intervals to know the voter preference. Surveying in which the sampling is done on successive occasions (over years, seasons, months, or weeks) according to the specified rule, with partial replacement of units is called successive (rotation) sampling. Beginning with the work of Jessen (1942) and followed by Patterson (1950), Eckler (1955), Rao and Graham (1964), Singh et al. (1992) and Singh (2005) among others have developed the theory of successive sampling. Feng and Zou (1997) and Biradar and Singh (2001) used the auxiliary information on both the occasions for estimating the current population mean in the successive sampling. Recently Singh and Vishwakarma (2007, 2009), Singh and Pal (2015 a, b, c, d) and Singh and Pal $(2016 \mathrm{a}, \mathrm{b}, \mathrm{c})$ have used the auxiliary information on both the occasion and envisaged several estimators for the estimating the population mean on current occasion in two-occasion successive (rotation) sampling. Motivated with the above work and utilizing the information on an auxiliary variable, readily available on the both occasions, we have proposed transformed estimators for estimating the current population mean in twooccasion successive (rotation) sampling. The procedure discussed in the above studies have used information only on the population mean $\bar{Z}$ of the auxiliary variable $z$, while in various survey situations information on other parameters of the auxiliary variable $z$ such as coefficient of variation $C_{z}$, population standard deviation $S_{z}$, population coefficients of skewness $\beta_{1}(z)$ and kurtosis $\beta_{2}(z)$; and the correlation coefficient $\rho_{y z}$ between study variable $y$ and auxiliary variable $z$; and the correlation coefficient $\rho_{x z}$ between the auxiliary variables $x$ and $z$ are known. The objective of the present paper is to propose a more precise estimator for estimating the population mean at current occasion in two occasions successive (rotation) sampling in the presence of auxiliary variable.

\section{Formulation And Notation Of The Proposed Estimator}

Let $U=\left(U_{1}, U_{2}, \ldots, U_{N}\right)$ be the finite population of size $N$ units, which has been sampled over two occasions. Let $x(y)$ be the variable under study on the first (second) occasion respectively. It is assumed that information on an auxiliary variable $z$ (stable over occasion) is readily available for the both the occasions. It is assumed that the population under investigation is large, and the sample size is constant on each occasion. A simple random sample of $n$ units is drawn without replacement $(W O R)$ on the first occasion. A random sub sample of $m(=n \lambda)$ units is retained (matched) from the sample drawn on the first occasion for its use on the current (second) occasion, while a fresh sample of size $u=(n-m)=n \mu$ units is drawn on the current (second) occasion, from the entire population by simple random sampling without replacement (SRSWOR) procedure so that the sample size on the current (second) is also $n$. The fractions of the matched and fresh samples are respectively designated by $\lambda$ and $\mu$ such that $\lambda+\mu=1$.

In what follows, we shall use the following notations throughout this paper.

$\bar{X}, \bar{Y}, \bar{Z}:$ The population means of the variables $x, y$ and $z$ respectively.

$\bar{x}_{m}, \bar{x}_{n}, \bar{y}_{u}, \bar{y}_{m}, \bar{z}_{u}, \bar{z}_{n}$ : The sample means of the respective variables based on the sample sizes indicated in suffices.

$C_{x}, C_{y}, C_{z}$ : The coefficients of variation of the variables $x, y$ and $z$ respectively,

$\rho_{y x}, \rho_{y z}, \rho_{x z}$ : The correlation coefficients between the variables shown in suffices .

$S_{x}^{2}=(N-1)^{-1} \sum_{i=1}^{N}\left(x_{i}-\bar{X}\right)^{2}, S_{y}^{2}=(N-1)^{-1} \sum_{i=1}^{N}\left(y_{i}-\bar{Y}\right)^{2}, S_{z}^{2}=(N-1)^{-1} \sum_{i=1}^{N}\left(z_{i}-\bar{Z}\right)^{2}$

are the population mean squares of $x, y$ and $z$ respectively, $f=n / N$ : Sampling fraction. 
To obtain the bias and mean square error $(M S E)$ of suggested class of estimators we define following quantities

$$
\begin{aligned}
& \bar{y}_{u}=\bar{Y}\left(1+e_{0 u}\right), \bar{y}_{m}=\bar{Y}\left(1+e_{0 m}\right), \bar{x}_{n}=\bar{X}\left(1+e_{1 n}\right), \bar{x}_{m}=\bar{X}\left(1+e_{1 m}\right), \bar{z}_{u}=\bar{Z}\left(1+e_{2 u}\right), \\
& \bar{z}_{n}=\bar{Z}\left(1+e_{2 n}\right), s_{y x(m)}=S_{y x}\left(1+e_{3 m}\right) \text { and } s_{x(m)}^{2}=S_{x}^{2}\left(1+e_{4 m}\right)
\end{aligned}
$$

such that

$$
E\left(e_{0 u}\right)=E\left(e_{0 m}\right)=E\left(e_{1 n}\right)=E\left(e_{1 m}\right)=E\left(e_{2 u}\right)=E\left(e_{2 n}\right)=E\left(e_{3 m}\right)=E\left(e_{4 m}\right)=0
$$

and

$$
\begin{aligned}
& E\left(e_{0 u}^{2}\right)=\left(\frac{1}{u}-\frac{1}{N}\right) C_{y}^{2}, E\left(e_{0 m}^{2}\right)=\left(\frac{1}{m}-\frac{1}{N}\right) C_{y}^{2}, E\left(e_{1 m}^{2}\right)=\left(\frac{1}{m}-\frac{1}{N}\right) C_{x}^{2}, E\left(e_{1 n}^{2}\right)=\left(\frac{1}{n}-\frac{1}{N}\right) C_{x}^{2}, \\
& E\left(e_{2 u}^{2}\right)=\left(\frac{1}{u}-\frac{1}{N}\right) C_{z}^{2}, E\left(e_{2 n}^{2}\right)=\left(\frac{1}{n}-\frac{1}{N}\right) C_{z}^{2}, E\left(e_{0 u} e_{0 m}\right)=-\frac{1}{N} C_{y}^{2}, E\left(e_{0 u} e_{1 m}\right)=-\frac{1}{N} \rho_{y x} C_{y} C_{x}, \\
& E\left(e_{0 u} e_{1 n}\right)=-\frac{1}{N} \rho_{y x} C_{y} C_{x}, E\left(e_{0 u} e_{2 u}\right)=\left(\frac{1}{u}-\frac{1}{N}\right) \rho_{y z} C_{y} C_{z}, E\left(e_{0 u} e_{2 n}\right)=-\frac{1}{N} \rho_{y z} C_{y} C_{z} \\
& E\left(e_{0 m} e_{1 m}\right)=\left(\frac{1}{m}-\frac{1}{N}\right) \rho_{y x} C_{y} C_{x}, E\left(e_{0 m} e_{1 n}\right)=\left(\frac{1}{n}-\frac{1}{N}\right) \rho_{y x} C_{y} C_{x}, E\left(e_{0 m} e_{2 u}\right)=-\frac{1}{N} \rho_{y z} C_{y} C_{z}, \\
& E\left(e_{0 m} e_{2 n}\right)=\left(\frac{1}{n}-\frac{1}{N}\right) \rho_{y z} C_{y} C_{z}, E\left(e_{1 m} e_{1 n}\right)=\left(\frac{1}{n}-\frac{1}{N}\right) \rho_{y x} C_{y} C_{x}, E\left(e_{1 m} e_{2 u}\right)=-\frac{1}{N} \rho_{x z} C_{x} C_{z}, \\
& E\left(e_{1 m} e_{2 n}\right)=\left(\frac{1}{n}-\frac{1}{N}\right) \rho_{x z} C_{x} C_{z}, E\left(e_{1 n} e_{2 u}\right)=-\frac{1}{N} \rho_{x z} C_{x} C_{z}, E\left(e_{1 n} e_{2 n}\right)=\left(\frac{1}{n}-\frac{1}{N}\right) \rho_{x z} C_{x} C_{z}, \\
& E\left(e_{2 u} e_{2 n}\right)=-\frac{1}{N} C_{z}^{2}, E\left(e_{1 m} e_{3 m}\right)=\frac{N(N-m)}{(N-1)(N-2)} \frac{\mu_{210}}{m \bar{X} S_{x y}}, E\left(e_{1 n} e_{3 m}\right)=\frac{N(N-n)}{(N-1)(N-2)} \frac{\mu_{210}}{m \bar{X} S_{x y}}, \\
& E\left(e_{4 m} e_{1 m}\right)=\frac{N(N-m)}{(N-1)(N-2)} \frac{\mu_{300}}{m \bar{X} S_{x}^{2}}, E\left(e_{4 m} e_{1 n}\right)=\frac{N(N-n)}{(N-1)(N-2)} \frac{\mu_{300}}{n \bar{X} S_{x}^{2}}, \\
& \mu_{r s t}=E\left[\left(x_{i}-\bar{X}\right)^{r}\left(y_{i}-\bar{Y}\right)^{s}\left(z_{i}-\bar{Z}\right)^{t}\right],(r, s, t) \geq 0 \text { are integers. }
\end{aligned}
$$

\section{Estimator Based On Unmatched Portion}

To estimate the population mean $\bar{Y}$ on the second (current) occasion an estimator based on a sample of size $u$ $=n \mu$ drawn, afresh on the second occasion is defined by

$$
\begin{aligned}
t_{u} & =\bar{y}_{u} \exp \left\{\frac{\delta\left(\bar{z}_{u}^{*}-\bar{Z}^{*}\right)}{\bar{z}_{u}^{*}+\bar{Z}^{*}}\right\}, \\
& =\bar{y}_{u} \exp \left\{\frac{\delta a\left(\bar{z}_{u}-\bar{Z}\right)}{a\left(\bar{z}_{u}+\bar{Z}\right)+2 b}\right\},
\end{aligned}
$$

where $\bar{Z}^{*}=a \bar{Z}+b, \bar{z}_{u}^{*}=a \bar{z}_{u}+b$ and $(a, b)$ are suitably chosen constants. The scalars $(a, b)$ may assume real values as well as parametric values $C_{z}$ (coefficient of variation of the auxiliary variable $z$ ), $\rho_{x z}$ (correlation coefficient between $x$ and $z$ ), $\beta_{1}(z)$ (coefficient of skewness of $z$ ), $\beta_{2}(z)$ (coefficient of kurtosis of $z$ ), $\bar{Z}$ (population mean of $z$ ) and $S_{z}$ (standard deviation $z$ ) etc for instance see Singh and Tailor (2003), Upadhyaya and Singh (1999) and Singh et al.(2004). 


\subsection{The Bias and MSE of Estimator $t_{u}$ Based on Unmatched Portion}

Expressing (2.1) in term of e's we have

$$
\begin{aligned}
t_{u} & =\bar{Y}\left(1+e_{0 u}\right) \exp \left\{\frac{\delta \theta e_{2 u}}{1+\theta e_{2 u}}\right\}, \\
& =\bar{Y}\left(1+e_{0 u}\right) \exp \left[\delta \theta e_{2 u}\left(1+\theta e_{2 u}\right)^{-1}\right],
\end{aligned}
$$

where

$$
\theta=a \bar{Z} /\{2(a \bar{Z}+b)\}
$$

We assume that $\left|\theta e_{2 u}\right|<1$, so that $\left(1+\theta e_{2 u}\right)^{-1}$ is expandable. Now expanding the right hand side of (3.2), multiplying out and neglecting terms e's having power greater than two we have

$$
t_{u} \cong \bar{Y}\left[1+e_{0 u}+\delta \theta e_{2 u}-\delta \theta e_{0 u} e_{2 u}+\frac{\delta \theta^{2}(\delta-2)}{2} e_{2 u}^{2}\right]
$$

or

$$
\left(t_{u}-\bar{Y}\right) \cong \bar{Y}\left[e_{0 u}+\delta \theta e_{2 u}-\delta \theta e_{0 u} e_{2 u}+\frac{\delta \theta^{2}(\delta-2)}{2} e_{2 u}^{2}\right] .
$$

Taking expectation on both sides of (3.3), we get the bias of proposed class of estimators $t_{u}$ up to first degree of approximation as

$$
B\left(t_{u}\right)=\left(\frac{1}{u}-\frac{1}{N}\right)\left(\frac{\bar{Y} \delta \theta}{2}\right)\left\{2 k_{y z}+\theta(\delta-2)\right\} C_{z}^{2},
$$

where $k_{y z}=\rho_{y z}\left(C_{y} / C_{z}\right)$.

Squaring both sides of (3.3) and neglecting terms of e's having power greater than two, we have

$$
\begin{aligned}
\left(t_{u}-\bar{Y}\right)^{2} & \cong \bar{Y}^{2}\left[e_{0 u}+\delta \theta e_{2 u}\right]^{2} \\
& \cong \bar{Y}^{2}\left[e_{0 u}^{2}+\delta^{2} \theta^{2} e_{2 u}^{2}+2 \theta e_{0 u} e_{2 u}\right] .
\end{aligned}
$$

Taking expectation on both sides of (3.5), we get the MSE of proposed class of estimators $t_{u}$ up to first degree of approximation as

$$
\operatorname{MSE}\left(t_{u}\right)=\bar{Y}^{2}\left(\frac{1}{u}-\frac{1}{N}\right)\left[C_{y}^{2}+\delta \theta C_{z}^{2}\left(\delta \theta+2 k_{y z}\right)\right] .
$$

Remark 3.1. Since $x$ and $y$ denote the same study variable over two occasions and $z$ is an auxiliary variable correlated to $x$ and $y$, therefore as mentioned in Reddy (1978), Cochran (1977) and Feng and Zou (1997), the coefficients of variation is a stable quantity; it can be assumed that the coefficients of variation of $x, y, z$ are considered to be approximately equal (i.e. $C_{y} \cong C_{x} \cong C_{z}$ ).

Thus putting $C_{y} \approx C_{z}$ in (3.4) and (3.6) we get the bias and MSE of $t_{u}$ to the first degree of approximation, respectively, as

$$
B\left(t_{u}\right)=\left(\frac{1}{u}-\frac{1}{N}\right)\left(\frac{\bar{Y} \delta \theta C_{y}^{2}}{2}\right)\left\{2 \rho_{y z}+\theta(\delta-2)\right\}
$$

and

$$
\operatorname{MSE}\left(t_{u}\right)=\left(\frac{1}{u}-\frac{1}{N}\right) S_{y}^{2}\left\{1+\delta \theta\left(\delta \theta+2 \rho_{y z}\right)\right\}
$$




$$
=\left(\frac{1}{u}-\frac{1}{N}\right) S_{y}^{2} \alpha_{3}
$$

where

$$
\alpha_{3}=\left\{1+\delta \theta\left(\delta \theta+2 \rho_{y z}\right)\right\} .
$$

\section{Estimator Based On Matched Portion}

Three chain-type estimators based on the sample of size $m(=n \lambda)$ common to both the occasions are defined by

$$
\begin{aligned}
t_{m 1} & =\bar{y}_{m}\left(\frac{\bar{x}_{n}}{\bar{x}_{m}}\right) \exp \left(\frac{\delta\left(\bar{z}_{n}^{*}-\bar{Z}^{*}\right)}{\bar{Z}^{*}+\bar{z}_{n}^{*}}\right), \\
& =\bar{y}_{m}\left(\frac{\bar{x}_{n}}{\bar{x}_{m}}\right) \exp \left(\frac{a \delta\left(\bar{z}_{n}-\bar{Z}\right)}{a\left(\bar{Z}+\bar{z}_{n}\right)+2 b}\right) \\
t_{m 2} & =\bar{y}_{m} \exp \left(\frac{\bar{x}_{n}-\bar{x}_{m}}{\bar{x}_{n}+\bar{x}_{m}}\right) \exp \left(\frac{\delta\left(\bar{z}_{n}^{*}-\bar{Z}^{*}\right)}{\bar{Z}^{*}+\bar{z}_{n}^{*}}\right), \\
& =\bar{y}_{m} \exp \left(\frac{\bar{x}_{n}-\bar{x}_{m}}{\bar{x}_{n}+\bar{x}_{m}}\right) \exp \left(\frac{a \delta\left(\bar{z}_{n}-\bar{Z}\right)}{a\left(\bar{Z}+\bar{z}_{n}\right)+2 b}\right) \\
t_{m 3} & =\left[\bar{y}_{m}+b_{y x(m)}\left(\bar{x}_{n}-\bar{x}_{m}\right)\right] \exp \left(\frac{\delta\left(\bar{z}_{n}^{*}-\bar{Z}^{*}\right)}{\bar{Z}^{*}+\bar{z}_{n}^{*}}\right), \\
& =\left[\bar{y}_{m}+b_{y x(m)}\left(\bar{x}_{n}-\bar{x}_{m}\right)\right] \exp \left(\frac{a \delta\left(\bar{z}_{n}-\bar{Z}\right)}{a\left(\bar{Z}+\bar{z}_{n}\right)+2 b}\right),
\end{aligned}
$$

where $b_{y x(m)}$ is the sample regression coefficient of $y$ on $x$ based on matched portion and $(a, b)$ are same as defined earlier.

\subsection{The Bias and MSE of Estimators Based on Matched Portion}

\subsubsection{Derivation of the bias and MSE of the class of estimators $t_{m 1}$}

Expressing (4.1) in term of e's we have

$$
\begin{aligned}
t_{m 1} & =\bar{Y}\left(1+e_{0 m}\right)\left(1+e_{1 n}\right)\left(1+e_{1 m}\right)^{-1} \exp \left\{\frac{\delta \theta e_{2 n}}{\left(1+\theta e_{2 n}\right)}\right\}, \\
& =\bar{Y}\left(1+e_{0 m}\right)\left(1+e_{1 n}\right)\left(1+e_{1 m}\right)^{-1} \exp \left(\delta \theta e_{2 n}\right)\left(1+\theta e_{2 n}\right)^{-1},
\end{aligned}
$$

We assume that $\left|e_{1 m}\right|<1$ and $\left|\theta e_{2 n}\right|<1$, so that $\left(1+e_{1 m}\right)^{-1}$ and $\left(1+\theta e_{2 u}\right)^{-1}$ are expandable. Now, expanding the right hand side of (4.4), multiplying out and neglecting terms of e's having power greater than two, we have

$$
\begin{aligned}
t_{m 1} & \cong \bar{Y}\left[1+e_{0 m}-\left(e_{1 m}-e_{1 n}\right)-\left(e_{0 m} e_{1 m}-e_{0 m} e_{1 n}\right)+\left(e_{1 m}^{2}-e_{1 m} e_{1 n}\right)\right. \\
& \left.+\delta \theta e_{2 n}+\delta \theta e_{0 m} e_{2 n}-\delta \theta\left(e_{1 m} e_{2 n}-e_{1 n} e_{2 n}\right)+\left\{\theta^{2} \delta(\delta-2) / 2\right\} e_{2 n}^{2}\right],
\end{aligned}
$$

or

$$
\left(t_{m 1}-\bar{Y}\right) \cong \bar{Y}\left[e_{0 m}-\left(e_{1 m}-e_{1 n}\right)-\left(e_{0 m} e_{1 m}-e_{0 m} e_{1 n}\right)+\left(e_{1 m}^{2}-e_{1 m} e_{1 n}\right)\right.
$$




$$
\left.+\delta \theta e_{2 n}+\delta \theta e_{0 m} e_{2 n}-\delta \theta\left(e_{1 m} e_{2 n}-e_{1 n} e_{2 n}\right)+\left\{\theta^{2} \delta(\delta-2) / 2\right\} e_{2 n}^{2}\right] .
$$

Taking expectation on both sides of (4.5), we get the bias of the proposed class of estimators $t_{m 1}$ up to first degree of approximation as

$$
B\left(t_{m 1}\right)=\bar{Y}\left[\left(\frac{1}{m}-\frac{1}{n}\right) C_{x}^{2}\left(1-k_{y x}\right)+\left(\frac{1}{n}-\frac{1}{N}\right)\left(\frac{\delta \theta C_{z}^{2}}{2}\right)\left(\delta+2 k_{y z}-2\right)\right] .
$$

Squaring both sides of (4.5) and neglecting terms of e's having power greater than two, we have

$$
\begin{aligned}
\left(t_{m 1}-\bar{Y}\right)^{2} & \cong \bar{Y}^{2}\left[e_{0 m}-\left(e_{1 m}-e_{1 n}\right)+\delta \theta e_{2 n}\right]^{2} \\
& \cong \bar{Y}^{2}\left[e_{0 m}^{2}+\left(e_{1 m}-e_{1 n}\right)^{2}+\delta^{2} \theta^{2} e_{2 n}^{2}\right. \\
& \left.-2\left(e_{0 m} e_{1 m}-e_{0 m} e_{1 n}\right)+2 \delta \theta e_{0 m} e_{2 n}-2 \delta \theta\left(e_{1 m} e_{2 n}-e_{1 n} e_{2 n}\right)\right] .
\end{aligned}
$$

Taking expectation on both sides of (4.7), we get the $M S E$ of the proposed class of estimators $t_{m 1}$ up to first degree of approximation as

$$
\operatorname{MSE}\left(t_{m 1}\right)=\bar{Y}^{2}\left[\left(\frac{1}{m}-\frac{1}{n}\right)\left\{C_{y}^{2}+C_{x}^{2}\left(1-2 k_{y x}\right)\right\}+\left(\frac{1}{n}-\frac{1}{N}\right)\left\{C_{y}^{2}+\delta \theta C_{z}^{2}\left(\delta \theta+2 k_{y z}\right)\right\}\right] .
$$

Under the assumption $C_{y} \cong C_{x} \cong C_{z}$, the expressions of bias and MSE of $t_{m 1}$ in (4.6) and (4.8) respectively reduce to:

$$
\begin{aligned}
& B\left(t_{m 1}\right)=\bar{Y} C_{y}^{2}\left[\left(\frac{1}{m}-\frac{1}{n}\right)\left(1-\rho_{y x}\right)+\left(\frac{1}{n}-\frac{1}{N}\right)\left(\frac{\delta \theta}{2}\right)\left(\delta+2 \rho_{y z}-2\right)\right] \\
& \operatorname{MSE}\left(t_{m 1}\right)=S_{y}^{2}\left[\frac{1}{m} \alpha_{1}+\frac{1}{n} \alpha_{2}-\frac{1}{N} \alpha_{3}\right],
\end{aligned}
$$

where

$$
\alpha_{1}=2\left(1-\rho_{y x}\right), \alpha_{2}=\left[-1+2 \rho_{y x}+\delta \theta\left(\delta \theta+2 \rho_{y z}\right)\right] \text { and } \alpha_{3}=\left(\alpha_{1}+\alpha_{2}\right)=\left[1+\delta \theta\left(\delta \theta+2 \rho_{y z}\right)\right] .
$$

\subsubsection{Derivation of the bias and MSE of the class of estimators $t_{m 2}$}

Expressing (4.2) in terms of e's we have

$$
\begin{aligned}
t_{m 2} & =\bar{Y}\left(1+e_{0 m}\right)\left\{-\frac{\left(e_{1 m}-e_{1 n}\right)}{2}\left(1+\frac{\left(e_{1 m}+e_{1 n}\right)}{2}\right)^{-1}\right\} \exp \left\{\delta \theta e_{2 n}\left(1+\theta e_{2 n}\right)^{-1}\right\} \\
& =\bar{Y}\left(1+e_{0 m}\right)\left(1-\frac{d_{m}}{2}+\frac{A_{m n}}{8}-\ldots\right)\left[1+\delta \theta e_{2 n}+\frac{\delta(\delta-2) \theta^{2}}{2} e_{2 n}^{2}+\ldots\right],
\end{aligned}
$$

where $d_{m}=\left(e_{1 m}-e_{1 n}\right)$ and $A_{m n}=\left(3 e_{1 m}^{2}-2 e_{1 m} e_{1 n}-e_{1 n}^{2}\right)$.

Expanding the right hand side of (4.19), multiplying out and neglecting terms of e's having power greater than two we have

or

$$
t_{m 2} \cong \bar{Y}\left[1+e_{0 m}-\frac{d_{m}}{2}-\frac{e_{0 m} d_{m}}{2}+\frac{A_{m n}}{8}+\delta \theta e_{2 n}+\delta \theta e_{0 m} e_{2 n}-\left(\frac{\delta \theta}{2}\right) d_{m} e_{2 n}+\frac{\delta(\delta-2) \theta^{2}}{2} e_{2 n}^{2}\right]
$$

$$
\left(t_{m 2}-\bar{Y}\right) \cong \bar{Y}\left[e_{0 m}-\frac{d_{m}}{2}-\frac{e_{0 m} d_{m}}{2}+\frac{A_{m n}}{8}+\delta \theta e_{2 n}+\delta \theta e_{0 m} e_{2 n}-\left(\frac{\delta \theta}{2}\right) d_{m} e_{2 n}+\frac{\delta(\delta-2) \theta^{2}}{2} e_{2 n}^{2}\right] .
$$


Taking expectation on both sides of (4.12), we get the bias of the proposed class of estimators $t_{m 2}$ to the first degree of approximation, as

$$
B\left(t_{m 2}\right)=\bar{Y}\left[\left(\frac{1}{m}-\frac{1}{n}\right) \frac{C_{x}^{2}}{8}\left(3-4 k_{y x}\right)+\left(\frac{1}{n}-\frac{1}{N}\right)\left(\frac{\delta \theta C_{z}^{2}}{2}\right)\left\{\theta(\delta-2)+2 k_{y z}\right\}\right] .
$$

Squaring both sides of (4.12) and neglecting terms of e's having power greater than two, we have

$$
\begin{aligned}
\left(t_{m 2}-\bar{Y}\right)^{2} & \cong \bar{Y}^{2}\left[e_{0 m}-\left(d_{m} / 2\right)+\delta \theta e_{2 n}\right]^{2} \\
& \cong \bar{Y}^{2}\left[e_{0 m}^{2}+\left(d_{m}^{2} / 4\right)+\delta^{2} \theta^{2} e_{2 n}^{2}-d_{m} e_{0 m}+2 \delta \theta e_{0 m} e_{2 n}-\delta \theta d_{m} e_{2 n}\right] .
\end{aligned}
$$

Taking expectation on both sides of (4.14), we get the MSE of the proposed class of estimators $t_{m 2}$ up to first degree of approximation as

$$
\begin{aligned}
\operatorname{MSE}\left(t_{m 2}\right)= & \left.\bar{Y}^{2}\left[\left(\frac{1}{m}-\frac{1}{N}\right) C_{y}^{2}+\left(\frac{1}{m}-\frac{1}{n}\right) \frac{C_{x}^{2}}{4}\left(1-4 k_{y x}\right)+\left(\frac{1}{n}-\frac{1}{N}\right) \delta \theta C_{z}^{2}\left(\delta \theta+2 k_{y z}\right)\right\}\right] \\
=\bar{Y}^{2}\left[\frac{1}{m}\left\{C_{y}^{2}+\frac{C_{x}^{2}}{4}\left(1-4 k_{y x}\right)\right\}\right. & +\frac{1}{n}\left\{\delta \theta C_{z}^{2}\left(\delta \theta+2 k_{y z}\right)-\frac{C_{x}^{2}}{4}\left(1-4 k_{y x}\right)\right\} \\
& \left.-\frac{1}{N}\left\{C_{y}^{2}+\delta \theta C_{z}^{2}\left(\delta \theta+2 k_{y z}\right)\right\}\right] .
\end{aligned}
$$

Under the assumption $C_{x} \cong C_{y} \cong C_{z}$, the bias and $M S E$ of $t_{m 2}$ in (4.3) and (4.15) respectively reduce to

$$
\begin{aligned}
& B\left(t_{m 2}\right)=\bar{Y} C_{y}^{2}\left[\left(\frac{1}{m}-\frac{1}{n}\right) \frac{1}{8}\left(3-4 \rho_{y x}\right)+\left(\frac{1}{n}-\frac{1}{N}\right)\left(\frac{\delta \theta}{2}\right)\left\{\theta(\delta-2)+2 \rho_{y z}\right\}\right] \\
& \operatorname{MSE}\left(t_{m}\right)=S_{y}^{2}\left[\frac{1}{m} \alpha_{1}^{*}+\frac{1}{n} \alpha_{2}^{*}-\frac{1}{N} \alpha_{3}\right],
\end{aligned}
$$

where

$$
\alpha_{1}^{*}=\left[1+(1 / 4)\left(1-4 \rho_{y x}\right)\right], \alpha_{2}^{*}=\left[\delta \theta\left(\delta \theta+2 \rho_{y z}\right)-(1 / 4)\left(1-4 \rho_{y x}\right)\right]
$$

and $\alpha_{3}=\left(\alpha_{1}^{*}+\alpha_{2}^{*}\right)=\left[1+\delta \theta\left(\delta \theta+2 \rho_{y z}\right)\right]$.

\subsubsection{Derivation of the bias and MSE of the class of estimators $t_{m 3}$}

Expressing (4.3) in terms of e's we have

$$
t_{m 3}=\left[\bar{Y}\left(1+e_{0 m}\right)-\beta_{y x} \bar{X}\left(1+e_{3 m}\right)\left(e_{1 m}-e_{1 n}\right)\left(1+e_{4 m}\right)^{-1}\right] \exp \left\{\frac{\delta \theta e_{2 n}}{\left(1+\theta e_{2 n}\right)}\right\},
$$

where $\beta_{y x}$ is the population regression coefficient of $y$ on $x$.

Expanding the right hand side of (4.18), multiplying out and neglecting terms of e's having power greater than two, we have

or

$$
\begin{aligned}
t_{m 3} & \cong \bar{Y}\left[1+e_{0 m}-k_{y x}\left(d_{m}+e_{3 m} d_{m}-e_{4 m} d_{m}\right)\right. \\
& \left.+\delta \theta e_{2 n}+\delta \theta e_{0 m} e_{2 n}-\delta \theta\left(e_{1 m} e_{2 n}-e_{1 n} e_{2 n}\right)+\left\{\theta^{2} \delta(\delta-2) / 2\right\} e_{2 n}^{2}\right],
\end{aligned}
$$

$$
\begin{aligned}
\left(t_{m 3}-\bar{Y}\right) & \cong \bar{Y}\left[e_{0 m}-k_{y x}\left(d_{m}+e_{3 m} d_{m}-e_{4 m} d_{m}\right)\right. \\
& \left.+\delta \theta e_{2 n}+\delta \theta e_{0 m} e_{2 n}-\delta \theta\left(e_{1 m} e_{2 n}-e_{1 n} e_{2 n}\right)+\left\{\theta^{2} \delta(\delta-2) / 2\right\} e_{2 n}^{2}\right] .
\end{aligned}
$$


Taking expectation on both sides of (4.19), we get the bias of the proposed class of estimators $t_{m 3}$ up to first degree of approximation as

$$
B\left(t_{m 3}\right)=-\bar{Y}\left[\left(\frac{1}{m}-\frac{1}{n}\right) \frac{N}{N-2} \frac{k_{y x}}{\bar{X}}\left(\frac{\mu_{210}}{\mu_{110}}-\frac{\mu_{300}}{\mu_{200}}\right)-\left(\frac{1}{n}-\frac{1}{N}\right)\left(\frac{\delta \theta C_{z}^{2}}{2}\right)\left\{\theta(\delta-2)+2 k_{y z}\right\}\right] .
$$

Squaring both sides of (4.19) and neglecting terms of e's having power greater than two, we have

$$
\begin{aligned}
\left(t_{m 3}-\bar{Y}\right)^{2} & \cong \bar{Y}^{2}\left[e_{0 m}-k_{y x} d_{m}+\delta \theta e_{2 n}\right]^{2} \\
& =\bar{Y}^{2}\left[e_{0 m}^{2}+k_{y x}^{2} d_{m}^{2}+\delta^{2} \theta^{2} e_{2 n}^{2}-2 k_{y x} e_{0 m} d_{m}+2 \delta \theta e_{0 m} e_{2 n}-2 k_{y x} \delta \theta d_{m} e_{2 n}\right] .
\end{aligned}
$$

Taking expectation on both sides of (4.21), we get the $M S E$ of the proposed class of estimators $t_{m 3}$ up to first degree of approximation as

$$
\begin{aligned}
\operatorname{MSE}\left(t_{m 3}\right) & =\bar{Y}^{2}\left[\left(\frac{1}{m}-\frac{1}{N}\right) C_{y}^{2}-\left(\frac{1}{m}-\frac{1}{n}\right) C_{x}^{2} k_{y x}^{2}+\left(\frac{1}{n}-\frac{1}{N}\right) \delta \theta C_{z}^{2}\left(\delta \theta+2 k_{y z}\right)\right] \\
& =\bar{Y}^{2}\left[\frac{1}{m} C_{y}^{2}\left(1-\rho_{y x}^{2}\right)+\frac{1}{n}\left\{\rho_{y x}^{2} C_{y}^{2}+\delta \theta C_{z}^{2}\left(\delta \theta+2 k_{y z}\right)\right\}-\frac{1}{N}\left\{C_{y}^{2}+\delta \theta C_{z}^{2}\left(\delta \theta+2 k_{y z}\right)\right\}\right]
\end{aligned}
$$

Under the assumption $C_{x} \cong C_{y} \cong C_{z}$, the $M S E$ of $t_{m 3}$ in (4.22) reduce to:

$$
\operatorname{MSE}\left(t_{m}\right)=S_{y}^{2}\left[\frac{1}{m} \alpha_{1}^{* *}+\frac{1}{n} \alpha_{2}^{* *}-\frac{1}{N} \alpha_{3}\right]
$$

where

$$
\alpha_{1}^{* *}=\left(1-\rho_{y x}^{2}\right), \alpha_{2}^{* *}=\left[\rho_{y x}^{2}+\delta \theta\left(\delta \theta+2 \rho_{y z}\right)\right],
$$

and $\alpha_{3}=\left(\alpha_{1}^{* *}+\alpha_{2}^{* *}\right)=\left[1+\delta \theta\left(\delta \theta+2 \rho_{y z}\right)\right]$.

\subsection{The Covariance between The Estimators of Matched Portion and Unmatched Portion}

The covariance between the estimator $t_{u}$ and the estimator $t_{m 1}$ is defined by

$$
\begin{aligned}
\operatorname{Cov}\left(t_{u}, t_{m 1}\right) & =E\left[\left(t_{u}-E\left(t_{u}\right)\right)\left(t_{m 1}-E\left(t_{m 1}\right)\right)\right] \\
& =E\left(t_{u}-\bar{Y}\right)\left(t_{m 1}-\bar{Y}\right) .
\end{aligned}
$$

Expressing $t_{u}$ and $t_{m 1}$ in terms of e's and neglecting terms of e's having power greater than two, we have

$$
\begin{aligned}
\operatorname{Cov}\left(t_{u}, t_{m 1}\right) & =\bar{Y}^{2} E\left[\left(e_{0 u}+\delta \theta e_{2 u}\right)\left(e_{0 m}-e_{1 m}+e_{1 n}+\delta \theta e_{2 n}\right)\right], \\
& =\bar{Y}^{2} E\left[e_{0 u} e_{0 m}-e_{0 u} e_{1 m}+e_{0 u} e_{1 n}+\delta \theta e_{0 u} e_{2 n}+\delta \theta e_{0 m} e_{2 u}-\delta \theta e_{2 u} e_{1 m}+\delta \theta e_{1 n} e_{2 u}+\delta^{2} \theta^{2} e_{2 u} e_{2 n}\right] \\
& =-\left(\bar{Y}^{2} / N\right)\left[C_{y}^{2}+\delta \theta\left(\delta \theta+2 k_{y z}\right) C_{z}^{2}\right] .
\end{aligned}
$$

Similarly, we can find the covariance between $\left(t_{u}\right.$ and $\left.t_{m 2}\right)$ and $\left(t_{u}\right.$ and $\left.t_{m 3}\right)$ as

$$
\operatorname{Cov}\left(t_{u}, t_{m 2}\right)=-\left(\bar{Y}^{2} / N\right)\left[C_{y}^{2}+\delta \theta\left(\delta \theta+2 k_{y z}\right) C_{z}^{2}\right]
$$

and

$$
\operatorname{Cov}\left(t_{u}, t_{m 3}\right)=-\left(\bar{Y}^{2} / N\right)\left[C_{y}^{2}+\delta \theta\left(\delta \theta+2 k_{y z}\right) C_{z}^{2}\right] .
$$

Under the assumption $C_{x} \cong C_{y} \cong C_{z}$, the expressions in (4.24), (4.25) and (4.26) respectively reduce to:

$$
\operatorname{Cov}\left(t_{u}, t_{m 1}\right)=-\left(S_{y}^{2} / N\right) \alpha_{3},
$$




$$
\operatorname{Cov}\left(t_{u}, t_{m 2}\right)=-\left(S_{y}^{2} / N\right) \alpha_{3},
$$

where $\alpha_{3}=\left[1+\delta \theta\left(\delta \theta+2 \rho_{y z}\right)\right]$.

\section{The Combined Estimator}

Combining the estimators $t_{u}$ and $t_{m i},(i=1,2,3)$ we have the ultimate estimator of the population mean $\bar{Y}$ as

$$
t_{i}=\omega_{i} t_{u}+\left(1-\omega_{i}\right) t_{m i},(i=1,2,3) ;
$$

where $\omega_{i}{ }^{\prime} s(i=1,2,3)$ are unknown constants to be determined under certain criterion. We note that for estimating the population mean on each occasion the estimator $t_{u}$ is suitable, which implies that more belief on $t_{u}$ could be observed by selecting $\omega_{i}(i=1,2,3)$ as 1 (or near to 1 ), while for estimating the change over the occasion, the estimators $t_{m i}(i=1,2,3)$ could be more suitable and hence $\omega_{i}$ might be selected as 0 (or near to 0 ). For asserting both the problems simultaneously, the appropriate (optimum) choices of $\omega_{i}$ are needed.

\section{Minimum MSEs of the Estimators $t_{i}(i=1,2,3)$.}

The mean squared errors of the estimators $t_{i}(i=1,2,3)$ to the first degree of approximation are given by

$$
\operatorname{MSE}\left(t_{i}\right)=\left[\omega_{i}^{2} \operatorname{MSE}\left(t_{u}\right)+\left(1-\omega_{i}\right)^{2} \operatorname{MSE}\left(t_{m i}\right)+2 \omega_{i}\left(1-\omega_{i}\right) \operatorname{Cov}\left(t_{u}, t_{m i}\right)\right],
$$

where $\operatorname{MSE}\left(t_{u}\right), \operatorname{MSE}\left(t_{m i}\right), \operatorname{Cov}\left(t_{u}, t_{m i}\right)(i=1,2,3)$ respectively given by (3.8), $\{(4.10),(4.17),(4.23)\}$ and $\{(4.27),(4.28),(4.29)\}$. Since the mean squared error of the estimators $t_{i}(i=1,2,3)$ in $(6.1)$ are the functions of unknown constants $\omega_{i}(i=1,2,3)$, therefore, we minimize $\operatorname{MSE}\left(t_{i}\right)(i=1,2,3)$ with respect to $\omega_{i}(i=1,2$, 3). Thus minimizing $\operatorname{MSE}\left(t_{i}\right)$ with respect $\omega_{i}$, we get the optimum values of $\omega_{i}$ as

$$
\omega_{\text {iopt }}=\frac{\left[\operatorname{MSE}\left(t_{m i}\right)-\operatorname{Cov}\left(t_{u}, t_{m i}\right)\right]}{\left[\operatorname{MSE}\left(t_{u}\right)+\operatorname{MSE}\left(t_{m i}\right)-2 \operatorname{Cov}\left(t_{u}, t_{m i}\right)\right]},(i=1,2,3) .
$$

Substitution of (6.2) in (6.1) yields the minimum $M S E$ of $t_{i}$ as

$$
\operatorname{Min.MSE}\left(t_{i}\right)=\frac{\left[\operatorname{MSE}\left(t_{u}\right) \operatorname{MSE}\left(t_{m i}\right)-\left\{\operatorname{Cov}\left(t_{u}, t_{m i}\right)\right\}^{2}\right]}{\left[\operatorname{MSE}\left(t_{u}\right)+\operatorname{MSE}\left(t_{m i}\right)-2 \operatorname{Cov}\left(t_{u}, t_{m i}\right)\right]},(i=1,2,3) .
$$

Further putting the values from equations (3.8), (4.10), (4.17), (4.23), (4.27), (4.28) and (4.29) in (6.2) and (6.3), the simplified values of $\omega_{\text {iopt }}$ and $\operatorname{Min} \cdot \operatorname{MSE}\left(t_{i}\right)$ are obtained as

$$
\begin{aligned}
& \omega_{1 \text { opt }}=\frac{\mu_{1}\left(\alpha_{3}-\mu_{1} \alpha_{2}\right)}{\left(\alpha_{3}-\mu_{1}^{2} \alpha_{2}\right)}, \\
& \operatorname{Min.} \operatorname{MSE}\left(t_{1}\right)=\frac{\alpha_{3}\left[(1-f) \alpha_{3}-\mu_{1} \alpha_{2}+\mu_{1}^{2} f \alpha_{2}\right] S_{y}^{2}}{n\left(\alpha_{3}-\mu_{1}^{2} \alpha_{2}\right)}, \\
& \omega_{2 o p t}=\frac{\mu_{2}\left(\alpha_{3}-\mu_{2} \alpha_{2}^{*}\right)}{\left(\alpha_{3}-\mu_{2}^{2} \alpha_{2}^{*}\right)}, \\
& \operatorname{Min.MSE}\left(t_{2}\right)=\frac{\alpha_{3}\left[(1-f) \alpha_{3}-\mu_{2} \alpha_{2}^{*}+\mu_{2}^{2} f \alpha_{2}^{*}\right] S_{y}^{2}}{n\left(\alpha_{3}-\mu_{2}^{2} \alpha_{2}^{*}\right)},
\end{aligned}
$$




$$
\begin{aligned}
& \omega_{3 o p t}=\frac{\mu_{3}\left(\alpha_{3}-\mu_{3} \alpha_{2}^{* *}\right)}{\left(\alpha_{3}-\mu_{3}^{2} \alpha_{2}^{* *}\right)}, \\
& \operatorname{Min.MSE}\left(t_{3}\right)=\frac{\alpha_{3}\left[(1-f) \alpha_{3}-\mu_{3} \alpha_{2}^{* *}+\mu_{3}^{2} f \alpha_{2}^{* *}\right] S_{y}^{2}}{n\left(\alpha_{3}-\mu_{3}^{2} \alpha_{2}^{* *}\right)},
\end{aligned}
$$

where $\mu_{i}{ }^{\prime} s(i=1,2,3)$ are the fractions of fresh samples to be drawn afresh on the current (second) occasion.

\subsection{Optimum Replacement Policy}

To obtain the optimum values of $\mu_{i}{ }^{\prime} s(i=1,2,3)$ so that the population mean $\bar{Y}$ may be estimated with maximum precision, we minimize the minimum MSEs of the estimator $t_{i}(i=1,2,3)$ given by $(6.5),(6.7)$ and (6.9) respectively with respect to $\mu_{i}{ }^{\prime} s(i=1,2,3)$ which result in quadratic equation in $\mu_{i}$ say $\hat{\mu}_{i}(i=1,2,3)$ are given below:

$$
\begin{aligned}
& \mu_{1}^{2} \alpha_{2}-2 \mu_{1} \alpha_{3}+\alpha_{3}=0, \\
& \hat{\mu}_{1}=\frac{\alpha_{3} \pm \sqrt{\alpha_{1} \alpha_{3}}}{\alpha_{2}}, \\
& \mu_{2}^{2} \alpha_{2}^{*}-2 \mu_{2} \alpha_{3}+\alpha_{3}=0, \\
& \hat{\mu}_{2}=\frac{\alpha_{3} \pm \sqrt{\alpha_{1}^{*} \alpha_{3}}}{\alpha_{2}^{*}} \\
& \mu_{3}^{2} \alpha_{2}^{* *}-2 \mu_{3} \alpha_{3}+\alpha_{3}=0, \\
& \hat{\mu}_{3}=\frac{\alpha_{3} \pm \sqrt{\alpha_{1}^{* *} \alpha_{3}}}{\alpha_{2}^{* *}} .
\end{aligned}
$$

From equations (6.11), (6.13) and (6.15), it is obvious that the real values of $\hat{\mu}_{i}(i=1,2,3)$ exist. If, the quantities under square roots are greater than or equal to zero (i.e. $\alpha_{1} \alpha_{3} \geq 0, \alpha_{1}^{*} \alpha_{3} \geq 0$ and $\alpha_{1}^{* *} \alpha_{3} \geq 0$ ). For any combination of correlations $\rho_{y x}$ and $\rho_{y z}$, and the scalars $\delta, a, b$ (and hence $\theta$ and $\delta$ ), which satisfy the condition of real situation, two real values of $\hat{\mu}_{i}(i=1,2,3)$ are possible. Hence, while selecting the values of $\hat{\mu}_{i}$, it should be observed that $0 \leq \hat{\mu}_{i} \leq 1$. Putting the admissible values of $\hat{\mu}_{i}$ say $\hat{\mu}_{i}^{(0)}$, from equations (6.11), (6.13) and (6.15) into equations (6.5), (6.7) and (6.9) respectively, we have the following optimum values of minimum mean squared error of the estimators $t_{i} \quad(i=1,2,3)$ :

$$
\begin{aligned}
& \operatorname{Min.MSE}\left(t_{1}\right)_{o p t}=\frac{\alpha_{3}\left[(1-f) \alpha_{3}-\hat{\mu}_{1}^{(0)} \alpha_{2}+\hat{\mu}_{1}^{(0) 2} f \alpha_{2}\right] S_{y}^{2}}{n\left(\alpha_{3}-\hat{\mu}_{1}^{(0) 2} \alpha_{2}\right)}, \\
& \operatorname{Min} \cdot \operatorname{MSE}\left(t_{2}\right)_{o p t}=\frac{\alpha_{3}\left[(1-f) \alpha_{3}-\hat{\mu}_{2}^{(0)} \alpha_{2}^{*}+\hat{\mu}_{2}^{(0) 2} f \alpha_{2}^{*}\right] S_{y}^{2}}{n\left(\alpha_{3}-\hat{\mu}_{2}^{(0) 2} \alpha_{2}^{*}\right)},
\end{aligned}
$$

and

$$
\operatorname{Min} \cdot \operatorname{MSE}\left(t_{3}\right)_{o p t}=\frac{\alpha_{3}\left[(1-f) \alpha_{3}-\hat{\mu}_{3}^{(0)} \alpha_{2}^{* *}+\hat{\mu}_{3}^{(0) 2} f \alpha_{2}^{* *}\right] S_{y}^{2}}{n\left(\alpha_{3}-\hat{\mu}_{3}^{(0) 2} \alpha_{2}^{* *}\right)}
$$




\section{Efficiency Comparisons}

The percent relative efficiencies of the proposed estimators ' $t_{i}$ ' $(i=1,2,3)$ with respect to (i) usual unbiased estimator $\bar{y}_{n}$, when there is no matching and (ii) traditional successive sampling estimator $\hat{\bar{Y}}=\varphi \bar{y}_{u}+(1-\varphi) \bar{y}_{d m}$ when no auxiliary information is used at any occasion, where $\bar{y}_{d m}=\bar{y}_{m}+\beta_{y x}\left(\bar{x}_{n}-\bar{x}_{m}\right)$ have been computed for different choices of $\rho_{y x}, \rho_{y z}$ and $\delta=-1$. Since, $\bar{y}_{n}$ and $\hat{\bar{Y}}$ are unbiased estimators of population mean $\bar{Y}$, therefore, following Sukhatme et al. (1984), the variance of usual unbiased estimator $\bar{y}_{n}$ and optimum variance of $\bar{y}_{\varphi}$ are respectively given by

$$
\operatorname{Var}\left(\bar{y}_{n}\right)=(1-f) \frac{S_{y}^{2}}{n},
$$

and

$$
\operatorname{Var}(\hat{\bar{Y}})_{o p t}=\left[\left(1+\sqrt{\left(1-\rho_{y x}^{2}\right)}\right)-2 f\right] \frac{S_{y}^{2}}{2 n},
$$

We have computed the percent relative efficiencies of $E_{i}^{(1)}$ and $E_{i}^{(2)}(i=1,2,3)$ of the proposed estimator ' $t_{i}$ ' $(i=1,2,3)$ with respect to the usual unbiased estimator $\bar{y}_{n}$ and $\hat{\bar{Y}}$ by using the following formulae:

$$
E_{i}^{(1)}=\frac{V\left(\bar{y}_{n}\right)}{\operatorname{Min} \cdot \operatorname{MSE}\left(t_{i}\right)_{\text {opt }}} \times 100
$$

and

$$
E_{i}^{(2)}=\frac{V(\hat{\bar{Y}})_{o p t}}{\min \cdot \operatorname{MSE}\left(t_{i}\right)_{\text {opt }}^{*}} \times 100 .
$$

Findings are shown in Table 7.1.

Table7.1. The PREs of $t_{i}(i=1,2,3)$ with respect to $\bar{y}_{n}$ and $\hat{\bar{Y}}$ for different values of $\theta, \rho_{y x}$ and $\rho_{y z}$.

\begin{tabular}{cccccccccccc}
\hline$\theta$ & $\rho_{y x}$ & $\rho_{y z}$ & $\mu_{1}^{(0)}$ & $E_{1}^{(1)}$ & $E_{1}^{(2)}$ & $\mu_{2}^{(0)}$ & $E_{2}^{(1)}$ & $E_{2}^{(2)}$ & $\mu_{3}^{(0)}$ & $E_{3}^{(1)}$ & $E_{3}^{(2)}$ \\
\hline 0.2 & 0.55 & 0.50 & 0.4914 & 116.77 & 106.08 & 0.5228 & 125.10 & 113.65 & 0.5232 & 125.22 & 113.76 \\
& & 0.55 & 0.4884 & 118.81 & 107.93 & 0.5198 & 127.33 & 115.67 & 0.5202 & 127.45 & 115.78 \\
& & 0.60 & 0.4853 & 120.92 & 109.85 & 0.5167 & 129.65 & 117.78 & 0.5171 & 129.78 & 117.89 \\
& & 0.65 & 0.4821 & 123.13 & 111.86 & 0.5135 & 132.07 & 119.98 & 0.5140 & 132.20 & 120.09 \\
& 0.70 & 0.4789 & 125.43 & 113.95 & 0.5103 & 134.59 & 122.27 & 0.5107 & 134.72 & 122.39 \\
& 0.60 & 0.50 & 0.5061 & 120.66 & 107.26 & 0.5320 & 127.58 & 113.40 & 0.5339 & 128.09 & 113.86 \\
& & 0.55 & 0.5031 & 122.79 & 109.15 & 0.5290 & 129.86 & 115.43 & 0.5309 & 130.39 & 115.91 \\
& & 0.60 & $*$ & - & - & 0.5259 & 132.24 & 117.55 & 0.5279 & 132.79 & 118.03 \\
& & 0.65 & 0.4968 & 127.30 & 113.16 & 0.5228 & 134.73 & 119.76 & 0.5247 & 135.28 & 120.25 \\
& 0.70 & 0.4936 & 129.71 & 115.30 & 0.5195 & 137.32 & 122.06 & 0.5215 & 137.89 & 122.57 \\
& 0.65 & 0.50 & 0.5228 & 125.10 & 108.42 & 0.5420 & 130.25 & 112.88 & 0.5467 & 131.53 & 113.99 \\
& & 0.55 & 0.5198 & 127.33 & 110.35 & 0.5390 & 132.60 & 114.92 & 0.5437 & 133.91 & 116.05 \\
& & 0.60 & 0.5167 & 129.65 & 112.36 & 0.5359 & 135.05 & 117.04 & 0.5406 & 136.39 & 118.20 \\
& & 0.65 & 0.5135 & 132.07 & 114.46 & 0.5327 & 137.60 & 119.25 & 0.5375 & 138.98 & 120.44 \\
0.3 & 0.70 & 0.5103 & 134.59 & 116.64 & 0.5295 & 140.27 & 121.56 & 0.5343 & 141.68 & 122.78 \\
\hline & 0.55 & 0.50 & 0.4837 & 122.02 & 110.84 & 0.5151 & 130.85 & 118.87 & 0.5156 & 130.97 & 118.98 \\
& & 0.55 & 0.4789 & 125.43 & 113.95 & 0.5103 & 134.59 & 122.27 & 0.5107 & 134.72 & 122.39 \\
& & 0.60 & 0.4739 & 129.07 & 117.25 & 0.5052 & 138.58 & 125.89 & 0.5057 & 138.72 & 126.02 \\
& & 0.65 & 0.4686 & 132.97 & 120.79 & $*$ & - & - & 0.5004 & 143.00 & 129.90 \\
& & 0.70 & 0.4632 & 137.14 & 124.58 & 0.4945 & 147.44 & 133.94 & 0.4950 & 147.59 & 134.07
\end{tabular}




\begin{tabular}{|c|c|c|c|c|c|c|c|c|c|c|c|}
\hline$\theta$ & $\rho_{y x}$ & $\rho_{y z}$ & $\mu_{1}^{(0)}$ & $E_{1}^{(1)}$ & $E_{1}^{(2)}$ & $\mu_{2}^{(0)}$ & $E_{2}^{(1)}$ & $E_{2}^{(2)}$ & $\mu_{3}^{(0)}$ & $E_{3}^{(1)}$ & $E_{3}^{(2)}$ \\
\hline & \multirow[t]{5}{*}{0.60} & 0.50 & 0.4984 & 126.14 & 112.12 & 0.5244 & 133.47 & 118.64 & 0.5263 & 134.02 & 119.13 \\
\hline & & 0.55 & 0.4936 & 129.71 & 115.30 & 0.5195 & 137.32 & 122.06 & 0.5215 & 137.89 & 122.57 \\
\hline & & 0.60 & 0.4886 & 133.51 & 118.68 & 0.5145 & 141.42 & 125.70 & 0.5164 & 142.01 & 126.23 \\
\hline & & 0.65 & 0.4833 & 137.58 & 122.29 & 0.5093 & 145.80 & 129.60 & 0.5112 & 146.42 & 130.15 \\
\hline & & 0.70 & 0.4778 & 141.94 & 126.17 & 0.5038 & 150.51 & 133.79 & 0.5057 & 151.16 & 134.36 \\
\hline & \multirow[t]{5}{*}{0.65} & 0.50 & 0.5151 & 130.85 & 113.40 & 0.5343 & 136.31 & 118.13 & 0.5391 & 137.67 & 119.31 \\
\hline & & 0.55 & 0.5103 & 134.59 & 116.64 & 0.5295 & 140.27 & 121.56 & 0.5343 & 141.68 & 122.78 \\
\hline & & 0.60 & 0.5052 & 138.58 & 120.10 & 0.5245 & 144.48 & 125.21 & 0.5293 & 145.95 & 126.49 \\
\hline & & 0.65 & $*$ & - & - & 0.5193 & 149.00 & 129.13 & 0.5240 & 150.53 & 130.45 \\
\hline & & 0.70 & 0.4945 & 147.44 & 127.78 & 0.5138 & 153.84 & 133.32 & 0.5186 & 155.44 & 134.71 \\
\hline \multirow[t]{15}{*}{0.4} & \multirow[t]{5}{*}{0.55} & 0.50 & 0.4789 & 125.43 & 113.95 & 0.5103 & 134.59 & 122.27 & 0.5107 & 134.72 & 122.39 \\
\hline & & 0.55 & 0.4721 & 130.34 & 118.41 & 0.5035 & 139.98 & 127.16 & 0.5040 & 140.11 & 127.28 \\
\hline & & 0.60 & 0.4650 & 135.72 & 123.29 & 0.4964 & 145.88 & 132.52 & 0.4968 & 146.02 & 132.65 \\
\hline & & 0.65 & 0.4575 & 141.63 & 128.66 & 0.4888 & 152.37 & 138.42 & 0.4892 & 152.53 & 138.56 \\
\hline & & 0.70 & 0.4495 & 148.17 & 134.60 & 0.4807 & 159.56 & 144.95 & 0.4812 & 159.73 & 145.10 \\
\hline & \multirow[t]{5}{*}{0.60} & 0.50 & 0.4936 & 129.71 & 115.30 & 0.5195 & 137.32 & 122.06 & 0.5215 & 137.89 & 122.57 \\
\hline & & 0.55 & 0.4868 & 134.84 & 119.85 & 0.5128 & 142.85 & 126.97 & 0.5147 & 143.45 & 127.51 \\
\hline & & 0.60 & 0.4797 & 140.45 & 124.85 & 0.5056 & 148.90 & 132.36 & 0.5076 & 149.54 & 132.92 \\
\hline & & 0.65 & 0.4721 & 146.63 & 130.34 & 0.4981 & 155.58 & 138.29 & $*$ & - & - \\
\hline & & 0.70 & 0.4641 & 153.48 & 136.42 & 0.4900 & 162.97 & 144.86 & 0.4919 & 163.68 & 145.50 \\
\hline & \multirow[t]{5}{*}{0.65} & 0.50 & 0.5103 & 134.59 & 116.64 & 0.5295 & 140.27 & 121.56 & 0.5343 & 141.68 & 122.78 \\
\hline & & 0.55 & 0.5035 & 139.98 & 121.31 & 0.5228 & 145.95 & 126.49 & 0.5275 & 147.44 & 127.78 \\
\hline & & 0.60 & 0.4964 & 145.88 & 126.42 & 0.5156 & 152.19 & 131.89 & 0.5204 & 153.76 & 133.25 \\
\hline & & 0.65 & 0.4888 & 152.37 & 132.05 & 0.5081 & 159.06 & 137.84 & 0.5128 & 160.72 & 139.29 \\
\hline & & 0.70 & 0.4807 & 159.56 & 138.28 & $*$ & - & - & 0.5048 & 168.44 & 145.97 \\
\hline \multirow[t]{15}{*}{0.5} & \multirow[t]{5}{*}{0.55} & 0.50 & 0.4772 & 126.62 & 115.02 & 0.5086 & 135.89 & 123.45 & 0.5091 & 136.03 & 123.57 \\
\hline & & 0.55 & 0.4686 & 132.97 & 120.79 & $*$ & - & - & 0.5004 & 143.00 & 129.90 \\
\hline & & 0.60 & 0.4594 & 140.09 & 127.26 & 0.4907 & 150.69 & 136.89 & 0.4912 & 150.84 & 137.03 \\
\hline & & 0.65 & 0.4495 & 148.17 & 134.60 & 0.4807 & 159.56 & 144.95 & 0.4812 & 159.73 & 145.10 \\
\hline & & 0.70 & 0.4387 & 157.40 & 142.99 & 0.4699 & 169.73 & 154.19 & 0.4703 & 169.91 & 154.35 \\
\hline & \multirow[t]{5}{*}{0.60} & 0.50 & 0.4919 & 130.95 & 116.40 & 0.5179 & 138.65 & 123.25 & 0.5198 & 139.23 & 123.76 \\
\hline & & 0.55 & 0.4833 & 137.58 & 122.29 & 0.5093 & 145.80 & 129.60 & 0.5112 & 146.42 & 130.15 \\
\hline & & 0.60 & 0.4741 & 145.03 & 128.92 & $*$ & - & - & 0.5019 & 154.51 & 137.34 \\
\hline & & 0.65 & 0.4641 & 153.48 & 136.42 & 0.4900 & 162.97 & 144.86 & 0.4919 & 163.68 & 145.50 \\
\hline & & 0.70 & 0.4533 & 163.14 & 145.02 & 0.4791 & 173.42 & 154.16 & 0.4811 & 174.20 & 154.84 \\
\hline & \multirow[t]{5}{*}{0.65} & 0.50 & 0.5086 & 135.89 & 117.77 & 0.5279 & 141.64 & 122.75 & 0.5326 & 143.07 & 123.99 \\
\hline & & 0.55 & * & - & - & 0.5193 & 149.00 & 129.13 & 0.5240 & 150.53 & 130.45 \\
\hline & & 0.60 & 0.4907 & 150.69 & 130.59 & 0.5100 & 157.27 & 136.30 & 0.5148 & 158.92 & 137.72 \\
\hline & & 0.65 & 0.4807 & 159.56 & 138.28 & $*$ & - & - & 0.5048 & 168.44 & 145.97 \\
\hline & & 0.70 & 0.4699 & 169.73 & 147.10 & 0.4891 & 177.43 & 153.77 & 0.4939 & 179.36 & 155.44 \\
\hline
\end{tabular}

Note: “*” indicates that $\mu_{i}^{(0)}$ does not exist.

\section{Discussions \& Conclusions}

Table 7.1 exhibits that:

(i) for fixed values of $\left(\theta, \rho_{y x}\right)$, the values of $\mu_{i}^{(0)}$ decreases, while the values of $E_{i}^{(1)}$ and $E_{i}^{(2)}(i=1,2,3)$ increase for increasing value of $\rho_{y z}$. This implies that if information on highly correlated auxiliary variable is available, increment in the precision of proposed estimators is obtained which reduce the cost of survey. Thus this type of situation is appreciable. Similar trends are obtained for fixed value of $\left(\rho_{y x}, \rho_{y z}\right)$ with varying the values of $\theta$. 
(ii) for fixed values of $\left(\theta, \rho_{y z}\right)$, the values of $\mu_{i}^{(0)}, E_{i}^{(1)}$ and $E_{i}^{(2)}(i=1,2,3)$ increase as the value of $\rho_{y x}$ increase. This behavior is in agreement with Sukhatme at al. (1984), results which explained that more the value of $\rho_{y x}$, more the fractions of fresh sample is needed at the current (second) occasion.

(iii) minimum values of $\mu_{1}^{(0)}=0.4387(\cong 0.44), \mu_{2}^{(0)}=0.4699(\cong 0.47)$ and $\mu_{1}^{(0)}=0.4703(\cong 0.47)$ which show that the fraction of fresh sample to be replaced is as low as about $44 \%$ (in case of estimator $t_{1}$ ) and $47 \%$ (in case of estimators $t_{2}$ and $t_{3}$ ) of the total sample size .

(iv) $E_{1}^{(j)}<E_{2}^{(j)}<E_{3}^{(j)}(j=1,2)$ which shows the superiority of the estimator $t_{3}$ over the estimators $t_{1}$ and $t_{2}$.

In general the values of $E_{i}^{(j)}$, s $(i=1,2,3 ; j=1,2)$ are greater than 100.Thus the proposed classes of estimators $t_{1}, t_{2}$ and $t_{3}$ are to be preferred over the usual unbiased estimator $\bar{y}_{n}$ and the natural estimator $\hat{\bar{Y}}$ in practice.

We conclude from the above discussions that the use of auxiliary information is extremely satisfying in terms of proposed estimators. It is also intelligible that if highly correlated auxiliary variable are used, moderately only a small fraction of sample on current occasion is preferred to be replaced by a fresh sample which reduce the cost of the survey.

\section{References}

1. A. R. Eckler, Rotation sampling. Ann. Math. Statist. 26(1955) 664-685.

2. G. N. Singh, On the use of chain type ratio estimator in successive Sampling. Statist. Transit. New series, 7(1) (2005) 21-26.

3. H. D. Patterson, Sampling on successive occasions with partial replacement of units. Jour. Roy. Statist. Assoc. 12 (B) (1950) 241- 255.

4. H. P. Singh and G. K. Vishwakarma, A general class of estimators in successive sampling. Metron 65(2) (2007) 201-227.

5. H. P. Singh and G. K. Vishwakarma, A general procedure for estimating population mean in successive sampling. Commun. Statist. Theo. Meth. 38(2009) 293-308.

6. H. P. Singh and R. Tailor, Use of known correlation coefficient in estimating the finite population mean. Statist. Transit. 6(4) (2003) 555-560.

7. H. P. Singh and S. K. Pal, An efficient effective rotation patterns in successive sampling over two occasions. Commun. Statist. The. Meth. 45(17) (2016b) 5017-5027.

8. H. P. Singh and S. K. Pal, Improved estimation of current population mean over two-occasions. Sri Lankan Jour. Appl. Statist. 16(1) (2015d) 1-19.

9. H. P. Singh and S. K. Pal, On the estimation of population mean in successive sampling. Int. Jour. Math. Sci. Appl. 5(1) (2015a) 179-185.

10. H. P. Singh and S. K. Pal, On the estimation of population mean incurrent occasions in two-occasion rotation patterns. Jour. Statist. Appl. Prob. 4(2) (2015c) 305-313.

11. H. P. Singh and S. K. Pal, On the estimation of population mean in rotation sampling. Jour. Statist. Appl. Pro. Lett. 2(1) (2015b) 331-336.

12. H. P. Singh and S. K. Pal, Search of good rotation patterns through exponential type regression estimator in successive sampling over two occasions. Ital. Jour. Pur. Appl. Math. 36(2016a) 567-582. 
13. H. P. Singh and S. K. Pal, Use of several auxiliary variables in estimating the population mean in a two occasion successive sampling. Commun. Statist. The. Meth. 45(23) (2016c) 6928-6942.

14. H. P. Singh H. P. Singh and V. P. Singh, A generalized efficient class of estimators of population mean in two-phase and successive Sampling. Int. Jour. Manage. Syst. 8(2) (1992)173-183.

15. H. P. Singh R. Tailor R. Tailor and M. S. Kakran, An improved estimator of population mean using power transformation. Jour. Ind. Soci. Agri. Statist. 58(2) (2004) 223-230.

16. J. N. K. Rao and J. E. Graham, Rotation design for sampling on repeated occasions. Jour. Amer. Statist. Assoc. 59 (1964) 492-509.

17. L.N. Upadhyaya and H. P. Singh, Use of transformed auxiliary variable in estimating the finite population mean. Biomet. Jour. 41 5(1999) 627-636.

18. P. V. Sukhatme B. V. Sukhatme S. Sukhatme and C. Ashok. Sampling theory of surveys with applications. Third Edition Ames IA Iowa State University Press (1984).

19. R. J. Jessen, Statistical investigation of a sample survey for obtaining form facts. Iowa Agricultural Experiment Station Road Bulletin No. 304 Ames USA (1942).

20. R. S. Biradar and H. P. Singh (2001), Successive sampling using auxiliary information on both the occasions. Calcutta Statist. Assoc. Bull. 51(2001) 243-251.

21. S. Feng and G. Zou, Sampling rotation method with auxiliary variable. Commun. Statist. Theo. Meth. 26(6) (1997)1497-1509.

22. V. N. Reddy, A study on the use of prior knowledge on certain population parameters in estimation. Sankhya 40(C) (1978) 29-37.

23. W.G. Cochran, Sampling Techniques. Third Edition Johan Wiley New York (1977). 